\title{
四苯基乙烯基共轭微孔聚合物的制备及其气体吸附
}

\author{
赵洋王笑颜张崇蒋加兴* \\ (陕西师范大学材料科学与工程学院 西安 710062)
}

\begin{abstract}
摘要 共轭微孔聚合物由于其在气体吸附与分离、非均相催化和光电子等领域的巨大应用前景而广受关注. 本文以四 苯基乙烯为基本构筑单元, 通过 Sonogashira-Hagihara 偶联反应制备了 3 种共轭微孔聚合物新材料, 研究了结构组成和 构建模块对制备聚合物孔性能和气体吸附性能的影响. 氮气吸附测试结果表明, 由 1,1,2,2-四炔四苯基乙烯自聚合制备 的 TPE-CMP1 具有较大的比表面积, 为 $1096 \mathrm{~m}^{2} \cdot \mathrm{g}^{-1}$. 在 $1.13 \mathrm{bar} / 273 \mathrm{~K}$ 条件下, TPE-CMP1 的 $\mathrm{CO}_{2}$ 吸附能力为 2.36 $\mathrm{mmol} \cdot \mathrm{g}^{-1}$; 在 $1.13 \mathrm{bar} / 77.3 \mathrm{~K}$ 条件下, TPE-CMP1 对 $\mathrm{H}_{2}$ 的吸附能力为 $1.35 \mathrm{wt} \%$. 另外, 制备的共轭微孔聚合物展示出较 高的 $\mathrm{CO}_{2} / \mathrm{N}_{2}$ 选择性吸附值. 由于这类多孔聚合物材料具有合成方法简单、优良的物理化学及热稳定性、高的比表面积 和 $\mathrm{CO}_{2}$ 吸附性能, 因此将在气体吸附与分离方面具有潜在的应用前景.

关键词 共轭微孔聚合物; 四苯基乙烯; Sonogashira-Hagihara 偶联反应; 比表面积; 气体吸附
\end{abstract}

\section{Conjugated Microporous Polymers Based on Tetraphenylethylene for Gas Adsorption}

\author{
Zhao, Yang Wang, Xiaoyan Zhang, Chong Jiang, Jia-Xing* \\ (School of Materials Science and Engineering, Shaanxi Normal University, Xi'an, Shaanxi 710062)
}

\begin{abstract}
Conjugated microporous polymers (CMPs) are attracting increasing attention due to their potential applications in areas such as gas adsorption, separation, heterogeneous catalysis and photoelectron. A series of CMPs based on tetraphenylethylene has been synthesized via Pd-catalyzed Sonogashira-Hagihara coupling reaction. During polymerization, all of the polymer networks precipitated from solution as yellow powders that are totally insoluble in common organic solvents tested because of their highly crosslinked structures. Thermogravimetric analysis indicated that all of the polymer networks are thermally stable up to $400{ }^{\circ} \mathrm{C}$ in nitrogen atmosphere. The high physicochemical and thermal stability could be attributed to the rigid nature of these aromatic polymers, composed solely of strong carbon-carbon and carbon-hydrogen bonds. The absence of the $\mathrm{C} \equiv \mathrm{H}$ stretching peaked at around $3280 \mathrm{~cm}^{-1}$ and the $\mathrm{C}-\mathrm{Br}$ stretching peaked at around $500 \mathrm{~cm}^{-1}$ in the FT-IR spectra for the polymer networks demonstrated that most of the ethynyl and bromine functional groups in the starting materials have been consumed by coupling reaction. Powder X-ray diffraction measurements revealed that all of the polymer networks are amorphous in nature. It was found that the homo-coupled polymer network of TPE-CMP1 from 1,1,2,2-tetrakis(4-ethynylphenyl)ethene shows the highest Brunauer-Emmet-Teller specific surface area up to $1096 \mathrm{~m}^{2} \cdot \mathrm{g}^{-1}$ among the resulting polymer networks. TPE-CMP1 exhibits a $\mathrm{CO}_{2}$ uptake ability of $2.36 \mathrm{mmol} \cdot \mathrm{g}^{-1}$ at 1.13 bar and $273 \mathrm{~K}$ with a $\mathrm{H}_{2}$ uptake capacity of $1.35 \mathrm{wt} \%$ at 1.13 bar and $77.3 \mathrm{~K}$. All of the polymer networks show high $\mathrm{CO}_{2} / \mathrm{N}_{2}$ selectivity around $30: 1$ and high isosteric heat of adsorption for $\mathrm{CO}_{2}$ up to $27.6 \mathrm{~kJ} \cdot \mathrm{mol}^{-1}$. Given the facile preparation strategy, the high physicochemical and thermal stability, the high surface area, and the outstanding $\mathrm{CO}_{2}$ sorption performances, these polymer networks are promising candidates for potential applications in post-combustion $\mathrm{CO}_{2}$ capture and sequestration technology.

Keywords conjugated microporous polymer; tetraphenylethylene; Sonogashira-Hagihara reaction; gas adsorption
\end{abstract}

\section{1 引言}

石油、煤、天然气等自然资源的不可持续性，以及 燃烧这些化石燃料释放大量二氧化碳所产生的温室效 应和环境污染, 导致全球性能源短缺和环境污染是当前 人类亟待解决的重大问题 ${ }^{[1]}$. 解决这些问题的可行方法
之一是开发能有效俘获二氧化碳或储存氢气的新材料 或技术. 有机多孔聚合物由于具有合成方法简单、合成 策略多样、高的比表面积、轻质、优良的物理化学及热 稳定性和易功能化修饰等特点 ${ }^{[2]}$, 因此是一类发展潜力 巨大的气体吸附材料. 过去十年间，一系列新型的有机 多孔聚合物材料如自具微孔聚合物 $(\mathrm{PIMs})^{[3,4]}$ 、超交联聚

\footnotetext{
*E-mail: jiaxing@snnu.edu.cn

Received January 30, 2015; published April 6, 2015.
}

Supporting information for this article is available free of charge via the Internet at http://sioc-journal.cn.

Project supported by the National Natural Science Foundation of China (No. 21304055) and the Fundamental Research Funds for the Central Universities (No. GK201501002).

项目受到国家自然科学(青年)基金(No. 21304055)和中央高校基本科研业务费专项基金(No. GK201501002)的支持. 
合物(HCPs) $)^{[5 \sim 8]}$ 、共价键合有机骨架聚合物 $(\mathrm{COFs})^{[9 \sim 12] 、}$

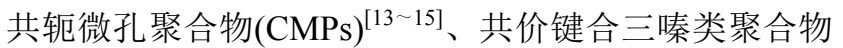

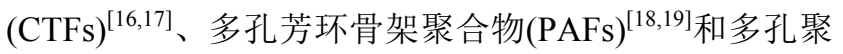
合物网络(PPNs) ${ }^{[20]}$ 等被相继开发出来.

2007 年, Cooper 等 ${ }^{[21}$ 通过 Sonogashira-Hagihara 偶 联反应首次合成了基于聚乙炔撑芳基(PAE)化合物的 CMPs, 其比表面积最高为 $834 \mathrm{~m}^{2} / \mathrm{g}$, 在 1 bar 压力和 77 $K$ 温度下, 吸氢能力为 $1.16 \mathrm{wt} \%$, 这是 CMPs 用于储氢 的第一个例子. 随后, 他们 ${ }^{[22]}$ 通过选用不同的聚合单体 合成了比表面积超过 $1000 \mathrm{~m}^{2} / \mathrm{g}$ 的 CMPs, 其吸氢能力达 到 1.4 wt\% (1 bar/77 K). 2010 年, Cooper 等 ${ }^{[23]}$ 又合成了 含螺旋结构的噻吩衍生物的 CMPs, 其比表面积达到 $1631 \mathrm{~m}^{2} / \mathrm{g}$, 在 $1 \mathrm{bar}$ 压力和 $77 \mathrm{~K}$ 温度下, 其吸氢能力提 高到 $1.72 \mathrm{wt} \%$. $\mathrm{Yu}$ 等 ${ }^{[24]}$ 报道了基于螺旋芴为骨架的 CMPs, 其比表面积为 $1043 \mathrm{~m}^{2} / \mathrm{g}$, 在 $60 \mathrm{bar}$ 压力和 $77 \mathrm{~K}$ 温度下, 其吸氢能力可达 $3.7 \mathrm{wt} \%$. 朱广山等 ${ }^{[18]}$ 通过镍 催化的 Yamamoto 聚合反应合成了具有四面体结构的有 机微孔聚合物材料 PAF-1, 其比表面积高达 $5640 \mathrm{~m}^{2} / \mathrm{g}$, 在 $1 \mathrm{bar}$ 压力和 $77 \mathrm{~K}$ 温度下的吸氢能力为 $1.50 \mathrm{wt} \%$. 韩 宝航等 ${ }^{[25]}$ 通过 Suzuki 和 Sonogashira-Hagihara 偶联反应 合成了螺旋芴基 CMPs, 比表面积为 $1020 \mathrm{~m}^{2} / \mathrm{g}$, 其对氢 气和二氧化碳的吸附能力分别为 $2.22 \mathrm{wt} \%(1 \mathrm{bar} / 77 \mathrm{~K})$ 和 $12.0 \mathrm{wt} \%(1 \mathrm{bar} / 273 \mathrm{~K})$; 随后, 他们 ${ }^{[26]}$ 通过 $\mathrm{FeCl}_{3}$ 氧化 聚合法合成了以聚咔唑为基本构建模块的 CMP, 其比 表面积为 $2220 \mathrm{~m}^{2} / \mathrm{g}$, 在 $1 \mathrm{bar} / 77 \mathrm{~K}$ 条件下, 制备聚合物 的吸氢能力高达 $2.8 \mathrm{wt} \%$, 其对二氧化碳的吸附能力也 提高到 $4.82 \mathrm{mmol} / \mathrm{g}(1 \mathrm{bar} / 273 \mathrm{~K})$. Cooper 等 ${ }^{[27]}$ 合成了一 系列羧基、氨基、甲基和羟基功能化的 CMPs, 功能化 的 CMPs 大大提高了与二氧化碳分子的吸附热, 其中羧 基功能化的 CMPs 对二氧化碳的吸附热达到 $32.6 \mathrm{~kJ}$ $\mathrm{mol}^{-1}$. 他们 ${ }^{[28]}$ 随后制备的三氰功能化的 CMPs 对二氧 化碳的吸附能力可达 $2.62 \mathrm{mmol} \cdot \mathrm{g}^{-1}$ (1 bar/273 K). 邓伟 侨等 ${ }^{[29]}$ 合成了钴/铝配位的 CMPs, 其在 $1 \mathrm{bar} / 298 \mathrm{~K}$ 条件 下对二氧化碳的吸附能力为 $1.80 \mathrm{mmol} \mathrm{g}^{-1}$. Qiao 等 ${ }^{[30]}$ 合成了氧化膦功能化的 CMPs, 其在 $1 \mathrm{bar} / 273 \mathrm{~K}$ 条件下 对二氧化碳的吸附能力为 $8.40 \mathrm{wt} \%$. Smaldone 等 ${ }^{[31]}$ 通过 Sonogashira 反应合成了以六苯并冦的 CMPs, 其在 1 bar/273 K 条件下对二氧化碳的吸附能力为 $9 \mathrm{wt} \%$. 最 近, Weber 等 ${ }^{[32]}$ 通过 Buchwald-Hartwig 偶联反应合成了 一系列三苯胺亚胺类 CMPs, 其在 $1 \mathrm{bar} / 273 \mathrm{~K}$ 条件下对 二氧化碳的吸附能力最高可达 $6.5 \mathrm{wt} \%$. 这些研究表明 共轭微孔聚合物在气体吸附领域具有巨大的应用前景. 然而, 相对于其它类型的有机微孔聚合物而言, 目前报 道的大部分共轭微孔聚合物无论是对氢气的储存能力 还是对二氧化碳的俘获能力都相对较低, 因此, 如何通 过选用合适的构建模块和聚合方法以提高共轭微孔聚 合物对气体的吸附性能成为近年来的研究热点.

本文以四炔四苯基乙烯为基本构建模块, 通过 Sonogashira-Hagihara 偶联反应制备了 3 种共轭微孔聚
合物新材料，其聚合物的合成路线如图 1 所示. 研究了 结构组成和构建模块对制备聚合物孔性能和气体吸附 性能的影响. 研究结果表明, 制备的聚合物具有良好的 热稳定性、物理化学稳定性、较高的比表面积和较窄的 孔尺寸及其分布. 由 1,1,2,2-四炔四苯基乙烯自聚合获 得的 TPE-CMP1 具有较大的比表面积, 为 $1096 \mathrm{~m}^{2} \cdot \mathrm{g}^{-1}$. 在 $1.13 \mathrm{bar} / 273 \mathrm{~K}$ 条件下, TPE-CMP1 的 $\mathrm{CO}_{2}$ 吸附能力 可达 $2.36 \mathrm{mmol} \cdot \mathrm{g}^{-1}$. 另外, 制备的共轭微孔聚合物展示 出较高的 $\mathrm{CO}_{2} / \mathrm{N}_{2}$ 选择性吸附数值.
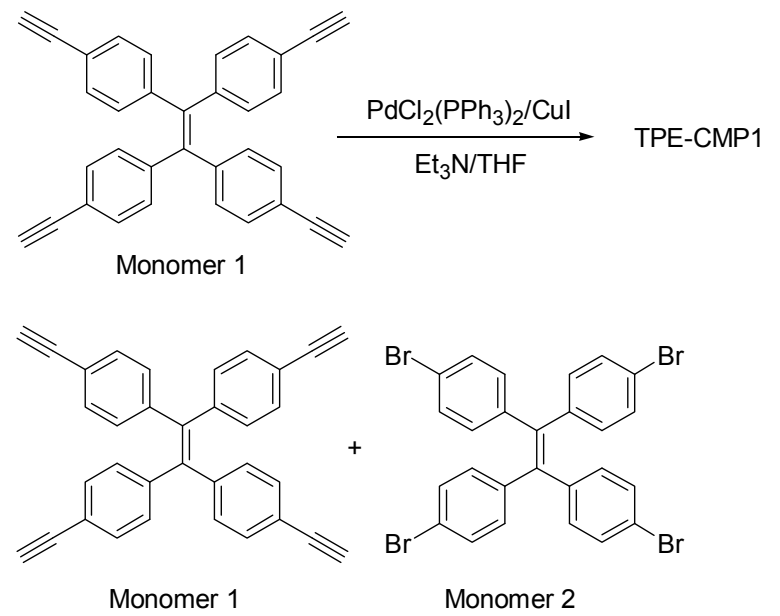

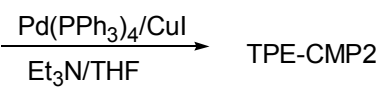
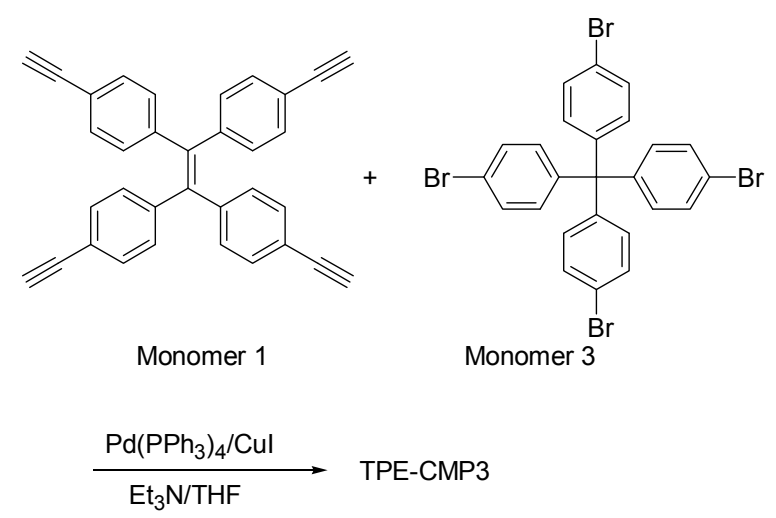

图 1 聚合物的合成路线

Figure 1 Synthetic routes for the polymer networks

\section{2 结果与讨论}

\section{1 红外光谱分析}

图 2 为所用单体及相应制备聚合物的红外光谱图. 在 1,1,2,2-四炔四苯基乙烯单体的红外光谱中, 端基 $\mathrm{C}$ $\mathrm{H}$ 在 $3280 \mathrm{~cm}^{-1}$ 的伸缩振动和 $\mathrm{C} \equiv \mathrm{C}$ 在 $2100 \mathrm{~cm}^{-1}$ 的伸缩 振动峰清晰可见, 而在制备聚合物的红外光谱中, 这两 处的特征峰完全消失, 表明在聚合反应过程中炔基功能 团的反应十分完全. 另外, 图 2 (b) (c)中单体 2 和单体 3 中端基 $\mathrm{C}-\mathrm{Br}$ 反应基团位于 512、532 和 $1078 \mathrm{~cm}^{-1}$ 的 
特征吸收峰也在聚合物 TPE-CMP2 和 TPE-CMP3 中的 红外光谱图上消失, 表明溴功能团在聚合反应中也反应 得十分彻底.
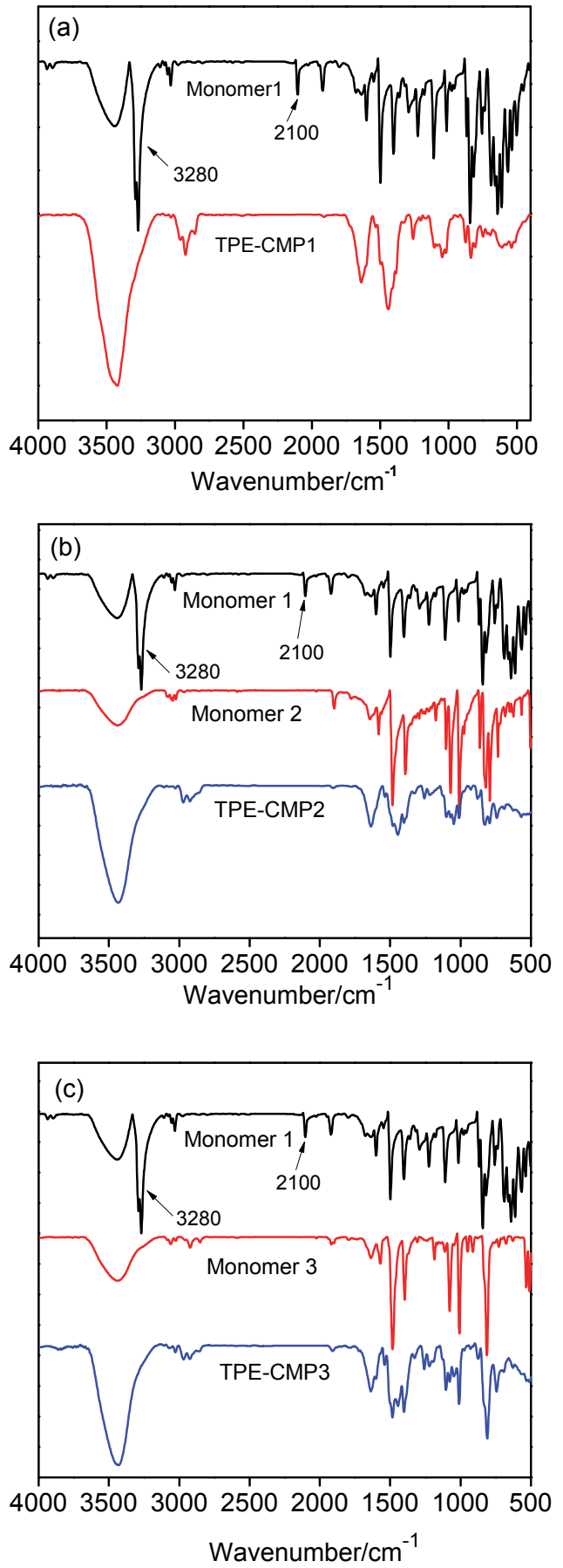

图 2 制备聚合物及单体的红外光谱图

Figure 2 FT-IR spectra for the monomers and polymer networks

\section{2 稳定性能分析}

制备聚合物的热稳定性由热重分析仪测定. 如图 3 所示, 在氮气环境下, 制备聚合物的热分解温度大约在 $400{ }^{\circ} \mathrm{C}$. 当温度为 $800{ }^{\circ} \mathrm{C}$ 时, TPE-CMP1 的失重大约为 20\%, TPE-CMP2 和 TPE-CMP3 的失重大约为 30\%, 表
明该类多孔聚合物材料具有良好的热稳定性. 另外，制 备的聚合物材料也展现出非常好的物理化学稳定性，在 乙醇、甲醇、二氯甲烷、丙酮和四氢呋喃等常用有机溶 剂中可稳定存在. 该类聚合物材料之所以表现出优良的 物理化学及热稳定性, 主要是因为该类聚合物都具有高 度交联和刚性的分子结构, 而且聚合物主链中只含有化 学键能较高的 $\mathrm{C}-\mathrm{C}$ 和 $\mathrm{C} \equiv \mathrm{C}$.

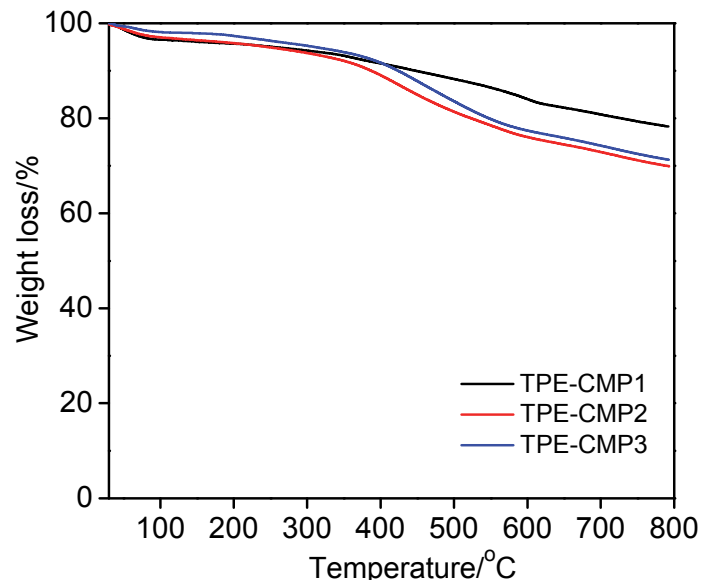

图 3 制备聚合物在氮气环境下的热重曲线(升温速率为 $10{ }^{\circ} \mathrm{C} / \mathrm{min}$ ) Figure 3 Thermogravimetric analysis trace for the polymer networks under a nitrogen atmosphere with a heating rate of $10{ }^{\circ} \mathrm{C} / \mathrm{min}$

\section{3 粉末 X 射线衍射和扫描电镜分析}

粉末 $\mathrm{X}$ 射线衍射测试表明制备聚合物在本质上都 属于无定形材料 (图 4), 这一结果与其它通过 Sonogashira 偶联反应获得的共轭微孔聚合物材料类 似 ${ }^{[21 ~ 33]}$. 在 $2 \theta$ 值 $28^{\circ}$ 左右的尖峰可能来自样品槽或祄 底. 另外，我们通过扫描电子显微镜对制备聚合物的表 面形貌进行了分析，如图 5 所示，制备聚合物材料基本 上都呈现出由纳米颗粒聚集而成的表面粗敉且不规则 的蜂窝状的表面形貌。

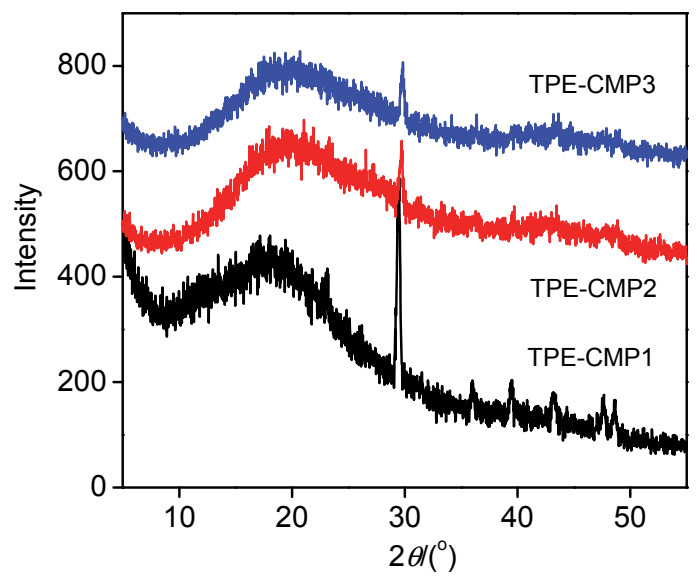

图 4 制备聚合物的粉末 X 射线衍射图

Figure 4 PXRD profile for the polymer networks 

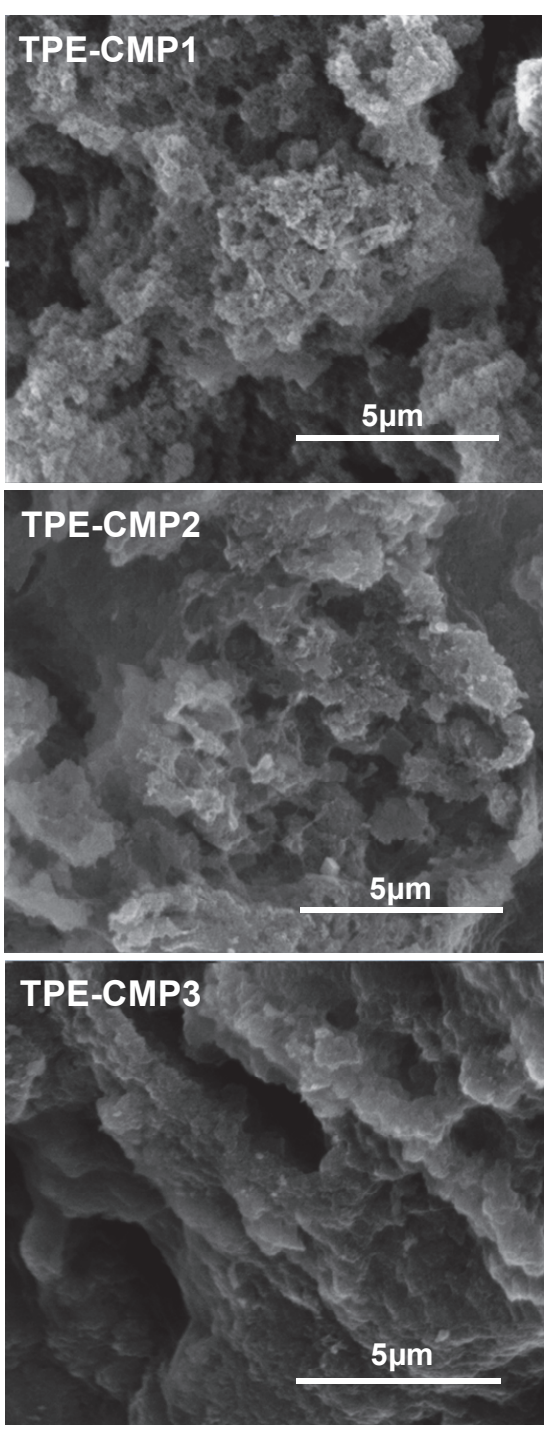

图 5 制备聚合物的扫描电镜照片

Figure 5 SEM images for the polymer networks

\section{4 孔性能分析}

制备聚合物的孔性能通过氮气吸附-脱附实验获得. 如图 6(a)所示, 制备的 3 种聚合物都表现出 I 型氮气吸 附特性, 在 $P / P_{0}=1 \times 10^{-5} \sim 1 \times 10^{-2}$ 的低压区, 氮气的 吸附量急剧上升, 表明制备聚合物中含有大量的微孔结 构. 而在相对压力 $P / P_{0}>0.8$ 时, 氮气吸附量也有少量 增加, 这可能是由于样品中颗粒间堆积形成的介孔和大 孔所致 ${ }^{[22]}$, 尤其是聚合物 TPE-CMP1, 在该区域氮气的 吸附量增加得较为明显, 表明该材料中含有更多的介孔 或大孔结构. 制备聚合物的氮气脱附曲线都存在较明显 的滞后现象, 这主要是因为气体吸附作用导致聚合物的 弹性形变和膨胀所致 ${ }^{[34]}$. 通过 Brunauer-Emmet-Teller (BET) 方法计算获得的比表面积分别为 TPE-CMP1: $1096 \mathrm{~m}^{2} \cdot \mathrm{g}^{-1}$; TPE-CMP2: $848 \mathrm{~m}^{2} \cdot \mathrm{g}^{-1}$; TPE-CMP3: 929 $\mathrm{m}^{2} \cdot \mathrm{g}^{-1}$. 制备聚合物的孔尺寸及其分布曲线通过非定域 密度泛函理论(NL-DFT)模型获得. 如图 6(b)所示, 制备
的聚合物主要由孔径为 1.1 和 $1.6 \mathrm{~nm}$ 左右的微孔组成, 另外还含有少量的介孔，其孔径大约在 2.1 和 $4.8 \mathrm{~nm}$ 左 右. 这表明制备聚合物的孔尺寸较窄且孔径分布比较集 中, 孔径分布基本上都集中在微孔部分，属于比较典型 的微孔材料. 相对于 TPE-CMP2 和 TPE-CMP3 而言, TPE-CMP1 的介孔含量相对较高, 这与其氮气吸附曲线 特性相一致。
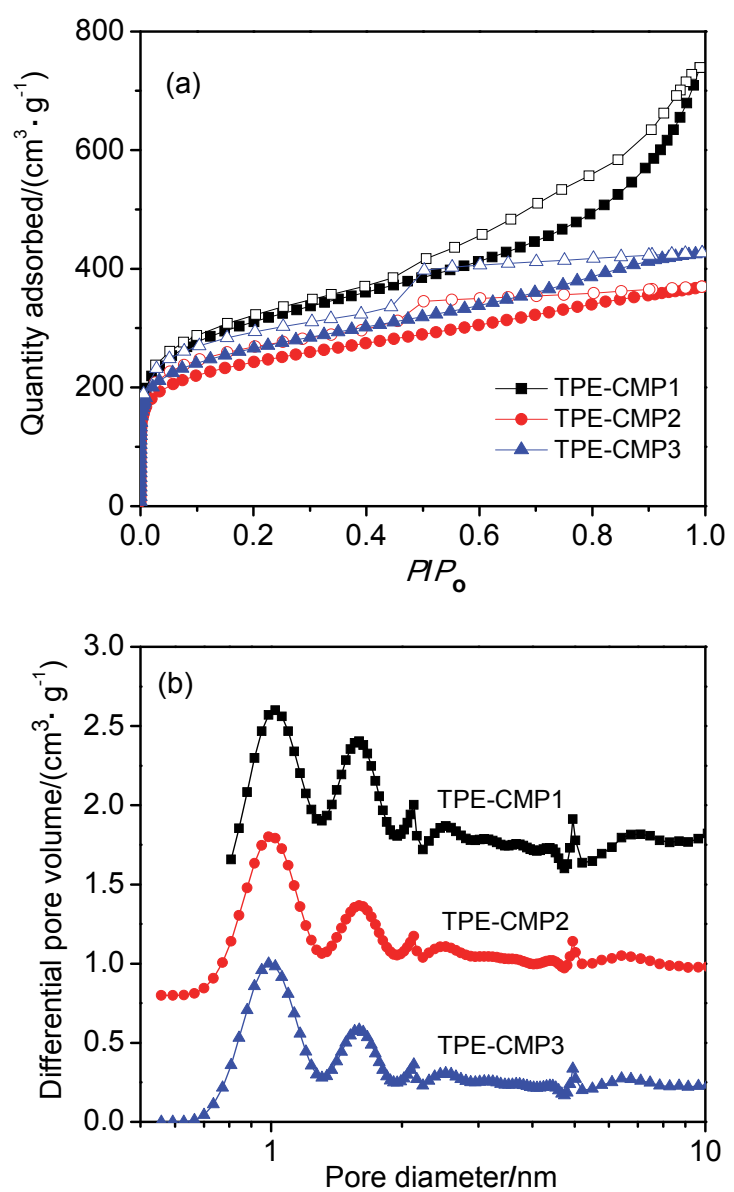

图 6 制备聚合物的 $\mathrm{N}_{2}$ 吸附-脱附等温曲线(a)和孔径分布曲线(b) $\begin{array}{llll}\text { Figure } 6 & \mathrm{~N}_{2} & \text { adsorption (filled symbols)-desorption (empty symbols) }\end{array}$ isotherms (a) and the pore size distribution curves (b) for the polymer networks

\section{5 气体吸附性能分析}

制备材料高的比表面积和微孔结构的特点将有利 于气体的吸附与分离. 因此我们测试了制备材料在不同 温度下对 $\mathrm{H}_{2} 、 \mathrm{CO}_{2} 、 \mathrm{CH}_{4}$ 及 $\mathrm{N}_{2}$ 的吸附性能，其测试结果 如表 1 所示. 图 7(a)是在 $77.3 \mathrm{~K} / 1.13 \mathrm{bar}$ 条件下测得的 制备聚合物的氢气吸附曲线. TPE-CMP1 在该条件下展 示出最高的氢气吸附能力 $(1.35 \mathrm{wt} \%)$, 这主要是因为在 制备的 3 个聚合物中, TPE-CMP1 具有最高的比表面积. 图 7(b)和 7(c)分别是在 273 和 $298 \mathrm{~K}$ 条件下获得的 $\mathrm{CO}_{2}$ 吸附曲线. 在 $273 \mathrm{~K} / 1.13 \mathrm{bar}$ 条件下, 制备聚合物对 $\mathrm{CO}_{2}$ 的吸附能力分别为 TPE-CMP1: $2.36 \mathrm{mmol} \cdot \mathrm{g}^{-1}$; TPE-CMP2: $2.15 \mathrm{mmol}^{\circ} \mathrm{g}^{-1}$; TPE-CMP3: $2.33 \mathrm{mmol} \cdot \mathrm{g}^{-1}$. 
虽然 TPE-CMP3 的比表面积略低于 TPE-CMP1, 但其对 $\mathrm{CO}_{2}$ 的吸附性能却与 TPE-CMP1 的相差不大, 这可能是 因为 TPE-CMP3 中主要是由孔尺寸小于 $2 \mathrm{~nm}$ 的微孔孔 结构组成, 而在 TPE-CMP1 中含有较多孔尺寸大于 2 $\mathrm{nm}$ 的介孔部分, 这与前面分析的氮气吸附曲线和孔尺 寸分布相一致. 在同等条件下, TPE-CMP1 的 $\mathrm{CO}_{2}$ 吸附 性能高于目前大部分报道的 CMPs 对 $\mathrm{CO}_{2}$ 的吸附性能, 例如羟基功能化的 CMP-1- $(\mathrm{OH})_{2}\left(1.80 \mathrm{mmol} \cdot \mathrm{g}^{-1}\right)^{[27]}$, 氨 基功能化的 CMP-1-AMDI (1.51 mmol•g $\left.{ }^{-1}\right)^{[35]}$ 和三蝶烯 CMP (2.13 mmol•g $\left.{ }^{-1}\right)^{[36]}$ 等, 虽然仍低于其它类型有机 微孔聚合物的 $\mathrm{CO}_{2}$ 吸附性能, 如 PPN-101 (5.1 mmol• $\left.\mathrm{g}^{-1}\right)^{[37]}$ 和 PPF-1 (6.1 mmol• $\left.\mathrm{g}^{-1}\right)^{[38]}$. 图 7(d) 是根据制备聚 合物在 273 和 $298 \mathrm{~K}$ 条件对 $\mathrm{CO}_{2}$ 吸附量计算获得的 $\mathrm{CO}_{2}$ 吸附热曲线. 制备聚合物都展示出较高的 $\mathrm{CO}_{2}$ 吸附热, 分别为 TPE-CMP1：27.6 $\mathrm{kJ}^{-} \mathrm{mol}^{-1}$; TPE-CMP2: 26.2 $\mathrm{kJ} \cdot \mathrm{mol}^{-1}$; TPE -CMP3: $24.2 \mathrm{~kJ} \bullet \mathrm{mol}^{-1}$. 另外, 通过采用亨 利方程和利用气体吸附曲线初始斜率的比值(图 S1-4),
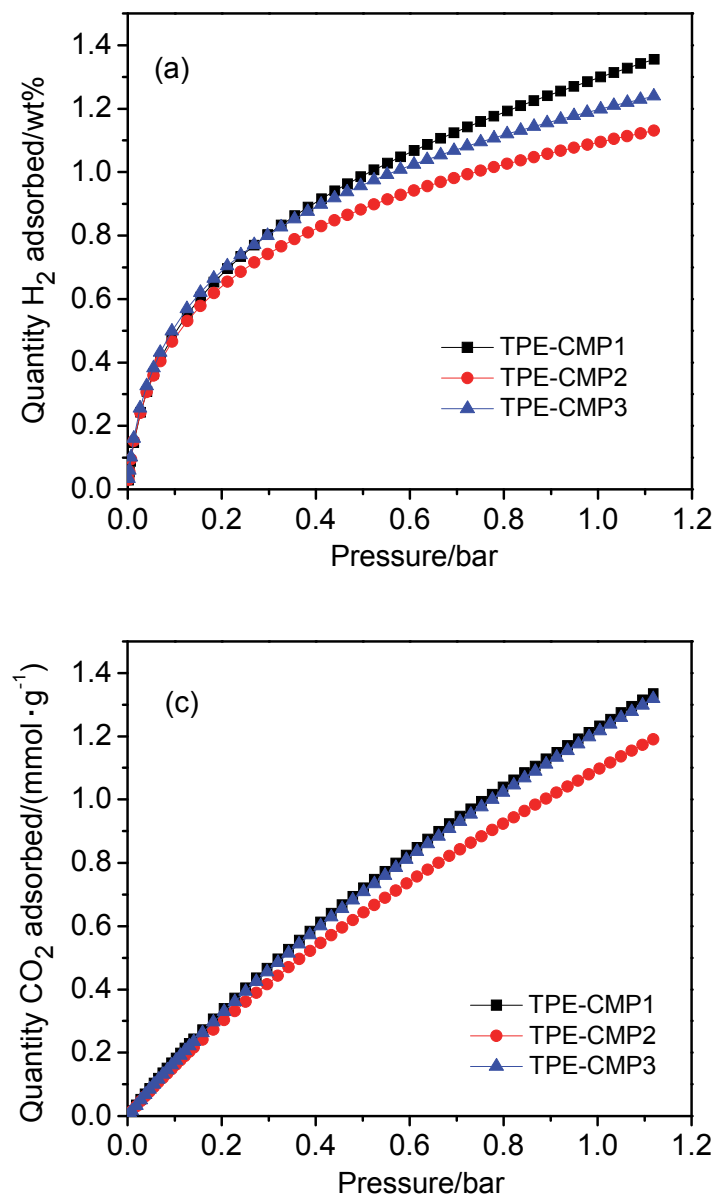

我们计算了制备聚合物对 $\mathrm{CO}_{2} / \mathrm{CH}_{4}$ 和 $\mathrm{CO}_{2} / \mathrm{N}_{2}$ 的选择性 吸附数值. 如表 1 所示, 制备聚合物对 $\mathrm{CO}_{2} / \mathrm{CH}_{4}$ 的选择 性吸附值大约为 4.5:1 左右, 而对 $\mathrm{CO}_{2} / \mathrm{N}_{2}$ 的选择性吸 附值可达 $30: 1$ 左右. 表明该类多孔聚合物材料具有较 好的 $\mathrm{CO}_{2} / \mathrm{N}_{2}$ 分离性能.

\section{3 结论}

选用具有四官能度可聚合基团的单体，以四苯基乙 烯为基本构建模块，通过 Sonogashira-Hagihara 偶联反 应一步法制备了一系列高比表面积的共轭微孔聚合物 材料. 制备的 TPE-CMP1 表现出较高的比表面积, 可达 $1096 \mathrm{~m}^{2} \cdot \mathrm{g}^{-1}$, 在 $273 \mathrm{~K} / 1.13 \mathrm{bar}$ 条件下, 其对 $\mathrm{CO}_{2}$ 的吸附 能力可达 $2.36 \mathrm{mmol} \cdot \mathrm{g}^{-1}$. 由于该类微孔聚合物材料具 有良好的物理化学及热稳定性、高的比表面积、合成方 法简单及良好的二氧化碳吸附性能，因此将在气体吸附 与分离方面具有潜在的应用前景.
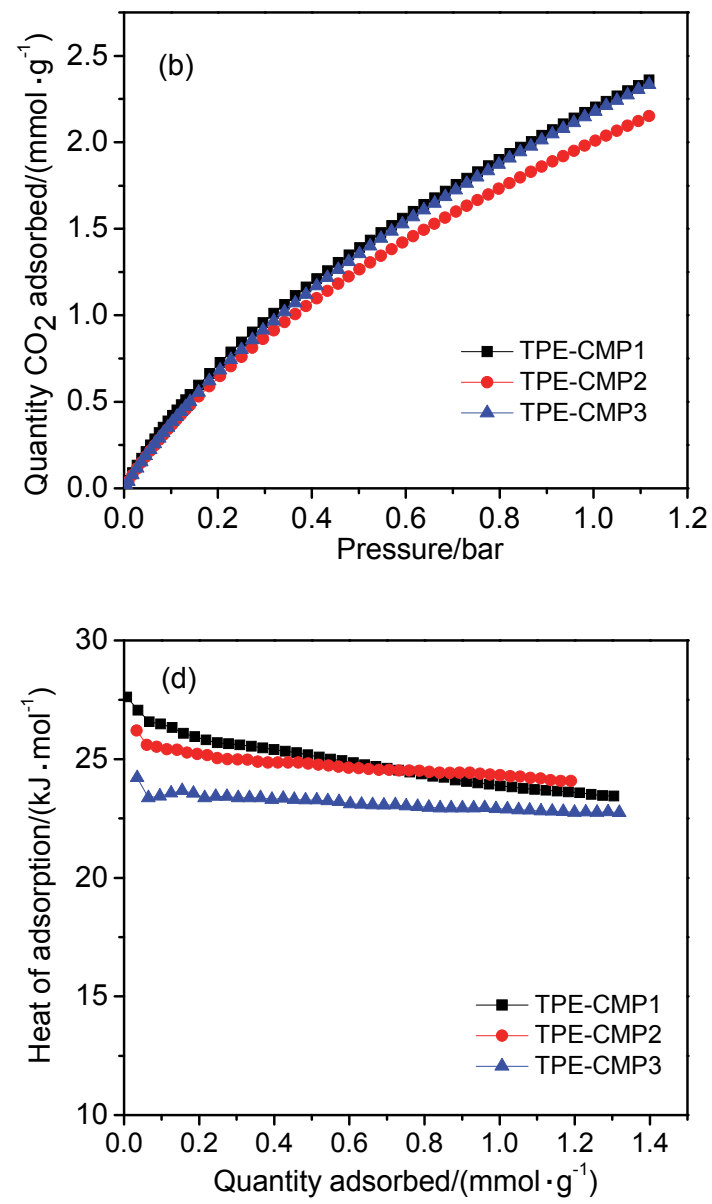

图 7 (a)制备聚合物在 $77.3 \mathrm{~K}$ 条件下的 $\mathrm{H}_{2}$ 吸附曲线; (b)在 $273 \mathrm{~K}$ 条件下的 $\mathrm{CO}_{2}$ 吸附曲线; (c)在 $298 \mathrm{~K}$ 条件下的 $\mathrm{CO}_{2}$ 吸附曲线; (d) 聚合物对 $\mathrm{CO}_{2}$ 的吸附热.

Figure 7 (a) $\mathrm{H}_{2}$ adsorption curves for the polymer networks at $77.3 \mathrm{~K}$ up to 1.13 bar. (b) $\mathrm{CO}_{2}$ adsorption isotherms at $273 \mathrm{~K}$ and (c) $298 \mathrm{~K}$. (d) Isosteric heats of adsorption for $\mathrm{CO}_{2}$ 
表 1 气体吸附及分离指数

Table 1 Gas uptake and selectivity

\begin{tabular}{|c|c|c|c|c|c|}
\hline \multirow{2}{*}{ Polymer } & \multirow{2}{*}{$\mathrm{H}_{2}$ uptake $^{a} / \mathrm{wt}^{0} \%$} & \multirow{2}{*}{$\mathrm{CH}_{4}$ uptake $^{b} /\left(\mathrm{mmol} \cdot \mathrm{g}^{-1}\right)$} & \multirow{2}{*}{$\mathrm{CO}_{2}$ uptake $^{b} /\left(\mathrm{mmol}_{\bullet} \mathrm{g}^{-1}\right)$} & \multicolumn{2}{|c|}{ Selectivity $^{c}$} \\
\hline & & & & $\mathrm{CO}_{2} / \mathrm{CH}_{4}$ & $\mathrm{CO}_{2} / \mathrm{N}_{2}$ \\
\hline TPE-CMP1 & 1.35 & 0.72 & 2.36 & 4.56 & 29.13 \\
\hline TPE-CMP2 & 1.31 & 0.67 & 2.15 & 4.46 & 29.03 \\
\hline TPE-CMP3 & 1.23 & 0.77 & 2.33 & 4.04 & 27.62 \\
\hline
\end{tabular}

${ }^{a}$ Data were obtained at 1.13 bar and $77.3 \mathrm{~K} .{ }^{b}$ Data were obtained at 1.13 bar and $273 \mathrm{~K} .{ }^{c}$ Adsorption selectivity based on the Henry's law.

\section{4 实验部分}

\subsection{1,1,2,2-四炔四苯基乙烯的合成}

1,1,2,2-四溴四苯基乙烯的制备：将四苯基乙烯 $(2.5 \mathrm{~g}, 7.5 \mathrm{mmol})$ 放入 $100 \mathrm{~mL}$ 单口瓶中, 然后将液溴 (3.75 mL, $0.75 \mathrm{mmol})$ 通过恒压滴液漏斗缓慢滴加入单 口瓶中, 滴加完后常温摚拌 $16 \mathrm{~h}$, 然后将热甲苯(100 $\mathrm{mL}$ )加入烧瓶中摚拌 $1 \mathrm{~h}$ 后, 将溶液减压蒸馏浓缩至 20 $\mathrm{mL}$ 左右后加入 $300 \mathrm{~mL}$ 正已烷进行搅拌约 $0.5 \mathrm{~h}$, 将产生 的沉淀过滤, 然后置于真空干燥箱内干燥 $24 \mathrm{~h}$ 获得白色 目标产物 $2.97 \mathrm{~g}($ 产率 $61.6 \%$ ).

1,1,2,2-四炔四苯基乙烯的制备：将 1,1,2,2-四溴四 苯基乙烯(4.1 g, $6.33 \mathrm{mmol}), \mathrm{PdCl}_{2}\left(\mathrm{PPh}_{3}\right)_{2}(0.226 \mathrm{~g}, 0.37$ $\mathrm{mmol}), \mathrm{CuI}(0.048 \mathrm{~g}, 0.25 \mathrm{mmol}), \mathrm{PPh}_{3}(0.2 \mathrm{~g}, 0.76 \mathrm{mmol})$, 置于 $100 \mathrm{~mL}$ 两口瓶中, 用氮气反复置换气体 3 次, 保证 反应环境在氮气气氛下进行. 用注射器将三乙胺 $\left(\mathrm{Et}_{3} \mathrm{~N}\right.$, $25 \mathrm{~mL})$ 注入反应瓶中, 再将三甲基硅烷乙炔(4.1 mL, $31.7 \mathrm{mmol}$ )注入反应瓶中, 加热到 $120{ }^{\circ} \mathrm{C}$, 摚拌反应 24 $\mathrm{h}$ 后, 将溶剂减压蒸干, 用 $\mathrm{CHCl}_{3}$ 溶解后, 用硅藻土过 滤, 滤液用 $\mathrm{Na}_{2}$ EDTA 溶液洗涤, 最后用 $\mathrm{Mg}_{2} \mathrm{SO}_{4}$ 干燥, 旋干, 然后进行柱分离提纯, 获得中间产物(4.2 g, 产率 $94 \%$ ). 将获得的中间产物(0.508 g, $0.71 \mathrm{mmol}$ )加入到 5 $\mathrm{mL}$ 甲醇的 $\mathrm{NaOH}(0.286 \mathrm{~g}, 7.1 \mathrm{mmol})$ 溶液中, 再加入 THF $(100 \mathrm{~mL})$, 室温搅拌 $6 \mathrm{~h}$ 后, 将溶剂减压蒸干, 用 $\mathrm{CHCl}_{3}$ 溶解, 然后水洗 3 次, 旋干, 用甲醇重结晶, 得到 黄色目标产物 1,1,2,2-四炔四苯基乙烯(0.273 g, 产率 95\%). ${ }^{1} \mathrm{H}$ NMR (400 MHz, $\mathrm{CDCl}_{3}$ ) (TMS) $\delta: 7.24$ (d, $J=$ $8.2 \mathrm{~Hz}, 8 \mathrm{H}), 6.93$ (d, $J=8.2 \mathrm{~Hz}, 8 \mathrm{H}), 3.07$ (s, 4H).

\section{2 聚合物的合成}

TPE-CMP1 的合成: 将 1,1,2,2-四炔四苯基乙烯 (0.22 g, $0.514 \mathrm{mmol}), \mathrm{PdCl}_{2}\left(\mathrm{PPh}_{3}\right)_{2}(15 \mathrm{mg}, 0.5 \mathrm{mmol})$, $\mathrm{CuI}(10 \mathrm{mg})$ 加入单口圆底反应瓶中, 然后用氮气反复置 换气体 3 次, 用注射器将三乙胺 $(4 \mathrm{~mL})$ 和 THF (4 mL)注 入反应瓶中. 加热到 $70{ }^{\circ} \mathrm{C}$, 搅拌反应 $48 \mathrm{~h}$ 后, 依次用 水、甲醇、二氯甲烷和丙酮洗涤并过滤, 最后将得到的 产物 TPE-CMP1 置于真空烘箱内真空干燥 24 h (102 mg, 产率 95\%).

TPE-CMP2 的合成: 该聚合中用到 1,1,2,2-四炔四 苯基乙烯( $0.107 \mathrm{~g}, 0.25 \mathrm{mmol}), 1,1,2,2$-四溴四苯基乙烯 (0.16 g, $0.25 \mathrm{mmol}), \mathrm{Pd}\left(\mathrm{PPh}_{3}\right)_{4}(15 \mathrm{mg})$ 和 $\mathrm{CuI}(10 \mathrm{mg})$, 其 合成方法与 TPE-CMP1 类似. 真空干燥后获得
TPE-CMP2 (172 mg, 产率 82\%).

TPE-CMP3 的合成: 该聚合中用到 1,1,2,2-四炔四 苯基乙烯( $0.107 \mathrm{~g}, 0.25 \mathrm{mmol}), 1,1,2,2$-四溴四苯基甲烷 $(0.156 \mathrm{~g}, 0.25 \mathrm{mmol}), \mathrm{Pd}\left(\mathrm{PPh}_{3}\right)_{4}(15 \mathrm{mg})$ 和 $\mathrm{CuI}(10 \mathrm{mg})$, 其合成步骤如 TPE-CMP1 类似. 真空干燥后获得 TPE-CMP3 (180 mg, 产率 86\%).

\section{References}

[1] Haszeldine, R. S. Science 2009, 325, 1647.

[2] Dawson, R.; Cooper, A. I.; Adams, D. J. Prog. Polym. Sci. 2012, 37, 530.

[3] Budd, P. M.; Ghanem, B. S.; Makhseed, S.; McKeown, N. B.; Msayib, K. J.; Tattershall, C. E. Chem. Commun. 2004, 230.

[4] McKeown, N. B.; Budd, P. M. Macromolecules 2010, 43, 5163.

[5] Lee, J. Y.; Wood, C. D.; Bradshaw, D.; Rosseinsky, M. J.; Cooper, A. I. Chem. Commun. 2006, 2670.

[6] Li, B.; Gong, R.; Wang, W.; Huang, X.; Zhang, W.; Li, H.; Hu, C.; Tan, B. Macromolecules 2011, 44, 2410.

[7] Xu, S.; Luo, Y.; Tan, B. Macromol. Rapid. Commun. 2013, 34, 471

[8] Luo, Y.; Li, B.; Wang, W.; Wu, K.; Tan, B. Adv. Mater. 2012, 24 5703

[9] Côté, A. P.; Benin, A. I.; Ockwig, N. W.; O'Keeffe, M.; Matzger, A. J.; Yaghi, O. M. Science 2005, 310, 1166.

[10] El-Kaderi, H. M.; Hunt, J. R.; Mendoza-Cortes, J. L.; Côté, A. P.; Taylor, R. E.; O'Keeffe, M.; Yaghi, O. M. Science 2007, 316, 268.

[11] Ding, S. Y.; Wang, W. Chem. Soc. Rev. 2013, 42, 548.

[12] Feng, X.; Ding, X. S.; Jiang, D. L. Chem. Soc. Rev. 2012, 41, 6010.

[13] Cooper, A. I. Adv. Mater. 2009, 21, 1291.

[14] Jiang, J. X.; Cooper, A. I. Top. Curr. Chem. 2010, 293, 1.

[15] Xu, Y.; Jin, S.; Xu, H.; Nagai, A.; Jiang, D. Chem. Soc. Rev. 2013, 42, 8012.

[16] Kuhn, P.; Antonietti, M.; Thomas, A. Angew. Chem., Int. Ed. 2008, 47,3450 .

[17] Ren, S. J.; Bojdys, M. J.; Dawson, R.; Laybourn, A.; Khimyak, Y. Z.; Adams, D. J.; Cooper, A. I. Adv. Mater. 2012, 24, 2357.

[18] Ben, T.; Ren, H.; Ma, S.; Cao, D.; Lan, J.; Jing, X.; Wang, W.; Xu, J.; Deng, F.; Simmons, J. M.; Qiu, S.; Zhu, G. Angew. Chem., Int. Ed. 2009, 48, 9457

[19] Yuan, Y.; Yan, Z.; Ren, H.; Liu, Q.; Zhu, G.; Sun, F. Acta Chim. Sinica 2012, 70, 1446. (元野, 间卓君, 任浩, 刘青英, 朱广山, 孙 福兴, 化学学报, 2012, 70, 1446.)

[20] Yuan, D.; Lu, W.; Zhao, D.; Zhou, H. C. Adv. Mater. 2011, 23, 3723.

[21] Jiang, J. X.; Su, F.; Trewin, A.; Wood, C. D.; Campbell, N. L.; Niu, H.; Dickinson, C.; Ganin, A. Y.; Rosseinsky, M. J.; Khimyak, Y. Z.; Cooper, A. I. Angew. Chem., Int. Ed. 2007, 46, 8574.

[22] Jiang, J. X.; Su, F.; Trewin, A.; Wood, C. D.; Niu, H.; Jones, J. T. A.; Khimyak, Y. Z.; Cooper, A. I. J. Am. Chem. Soc. 2008, 130, 7710.

[23] Jiang, J. X.; Laybourn, A.; Clowes, R.; Khimyak, Y. Z.; Bacsa, J.; Higgins, S. J.; Adams, D. J.; Cooper, A. I. Macromolecules 2010, 43, 7577.

[24] Yuan, S. W.; Kirklin, S.; Dorney, B.; Liu, D. J.; Yu, L. P. Macromolecules 2009, 42, 1554.

[25] Chen, Q.; Wang, J. X.; Wang, Q.; Bian, N.; Li, Z. H.; Yan, C. G.; Han, B. H. Macromolecules 2011, 44, 7987.

[26] Chen, Q.; Luo, M.; Hammershoj, P.; Zhou, D.; Han, Y.; Laursen, B. W.; Yan, C. G.; Han, B. H. J. Am. Chem. Soc. 2012, 134, 6084.

[27] Dawson, R.; Adams, D. J.; Cooper, A. I. Chem. Sci. 2011, 2, 1173.

[28] Ren, S.; Dawson, R.; Laybourn, A.; Jiang, J. X.; Khimyak, Y.; Adams, D. J.; Cooper, A. I. Polym. Chem. 2012, 3, 928. 
[29] Xie, Y.; Wang, T. T.; Liu, X. H.; Zou, K.; Deng, W. Q. Nat. Commun. 2013, 4, 1960.

[30] Qiao, S.; Huang, W.; Du, Z.; Chen, X.; Shieh, F. K.; Yang, R. New. J. Chem. 2015, 39, 136.

[31] Thompson, C. M.; Li, F.; Smaldone, R. A. Chem. Commun. 2014, 50,6171 .

[32] Liao, Y.; Weber, J.; Faul, C. F. Chem. Commun. 2014, 50, 8002.

[33] Zhang, T.; Wang, H.; Ma, H.; Sun, F.; Cui, X.; Zhu, G. Acta Chim. Sinica 2013, 71, 1598. (张婷婷, 王海涛, 马和平, 孙福兴, 崔小
强, 朱广山, 化学学报, 2013, 71, 1598.)

[34] Weber, J.; Antonietti, M.; Thomas, A. Macromolecules 2008, 41, 2880

[35] Ratvijitvech, T.; Dawson, R.; Laybourn, A.; Khimyak, Y. Z.; Adams, D. J.; Cooper, A. I. Polymer 2014, 55, 321.

[36] Zhang, C.; Zhai, T. L.; Wang, J. J.; Wang, Z.; Liu, J. M.; Tan, B.; Yang, X. L.; Xu, H. B. Polymer 2014, 55, 3642.

[37] Zhang, M.; Perry, Z.; Park, J.; Zhou, H. C. Polymer 2014, 55, 335.

[38] Zhu, Y.; Long, H.; Zhang, W. Chem. Mater. 2013, 25, 1630.

(Qin, X.) 